\title{
Modeling individual migraine severity with autoregressive ordered probit models
}

\author{
Claudia Czado ${ }^{1}$ Anette Heyn ${ }^{2}$ Gernot Müller ${ }^{3}$
}

\begin{abstract}
This paper considers the problem of modeling migraine severity assessments and their dependence on weather and time characteristics. We take on the viewpoint of a patient who is interested in an individual migraine management strategy. Since factors influencing migraine can differ between patients in number and magnitude, we show how a patient's headache calendar reporting the severity measurements on an ordinal scale can be used to determine the dominating factors for this special patient. One also has to account for dependencies among the measurements. For this the autoregressive ordinal probit (AOP) model of Müller and Czado (2005) is utilized and fitted to a single patient's migraine data by a grouped move multigrid Monte Carlo (GM-MGMC) Gibbs sampler. Initially, covariates are selected using proportional odds models. Model fit and model comparison are discussed. A comparison with proportional odds specifications shows that the AOP models are preferred.
\end{abstract}

Keywords: Bayes factor; Deviance; Ordinal valued time series; Markov Chain Monte Carlo (MCMC); Proportional odds; Regression;

\section{Introduction}

According to Prince et al. (2004) forty-five million Americans seek medical attention for head pain yearly causing an estimated labor cost of $\$ 13$ billion. They found in their study that about half of their migraine patients are sensitive to weather. However some studies investigating the relationship between weather conditions and headache have been negative or inclusive (see Prince et al. (2004) and Cooke et al. (2000) for specific references). In these studies the frequency of headache occurrences and the daily maximum or total score of an ordinal severity assessment have been the focus.

Here we focus directly on studying and modeling the observed severity categories collected using a headache calendar. In particular we want to investigate the four daily ratings of the

\footnotetext{
${ }^{1}$ Professor at Center for Mathematics, Munich University of Technology, Boltzmannstr. 3, 85747 Garching, Germany, e-mail: cczado@ma.tum.de, Phone: +4989289 17428, Fax: +498928917435

${ }^{2}$ Anette Heyn's part of the work was conducted at the Center for Mathematics, Munich University of Technology, while she worked on her diploma thesis

${ }^{3}$ Corresponding author. Postdoctoral Assistant, Center for Mathematics, Munich University of Technology, Boltzmannstr. 3, 85747 Garching, Germany, e-mail: mueller@ma.tum.de, Phone: +4989289 17441, Fax: +498928917435
} 
headache intensity obtained from a patient in study conducted by psychologist T. KosteckiDillon, Toronto, Canada, resulting in an ordinal valued time series. We take on the viewpoint of the patient who is interested in an individual migraine management strategy. Since factors influencing migraine can differ between patients in number and magnitude, we use the patient's headache calendar to determine the dominating factors for this patient.

Most studies ignore correlation among measurements on the same patient. We will show that this correlation can be very high and should not be ignored. For example, Prince et al. (2004) use daily maximum and total scores as response variable relating to factors obtained from a factor analysis of the weather data alone in a regression setup ignoring this correlation.

For studying headache occurrences Piorecky et al. (1996) used a generalized estimating approach (GEE) introduced by Zeger and Liang (1986) to adjust for the dependence between multiple measurements. While GEE could also be used for ordinal valued time series (see for example Liang et al. (1992), Heagerty and Zeger (1996) and Fahrmeir and Pritscher (1996)), we prefer a likelihood based regression time series approach to investigate the influence of weather conditions on migraine severity. One major reason for this preference is to have a complete statistical model specification, which allows the usage of standard model comparison techniques and forecasts in dynamic models.

Kauermann (2000) also considered the problem of modeling ordinal valued time series with covariates. He used a nonparametric smoothing approach by allowing for time varying coefficients in a proportional odds model. While Kauermann (2000) uses local estimation, Gieger (1997) and Fahrmeir et al. (1999) consider spline fitting within the GEE framework. Wild and Yee (1996) focus on smooth additive components. While these approaches are useful for fitting the data, a hierarchical time series approach which we propose here is easier to interpret and has the potential for forecasting. In particular, we will use an autoregressive ordered probit (AOP) model introduced by Müller and Czado (2005). It is based on a threshold approach using a latent real valued time series. It is fitted and validated in a Bayesian setting using Markov Chain Monte Carlo (MCMC) methods.

Since as already mentioned many studies investigating the relationship between headache and weather conditions have been inclusive, we believe management of migraine headaches should be tailored to the individual migraine sufferer. Since migraine headaches are a persistent problem such an individual analysis should be based on a headache calendar of the individual. Such an individual approach was also followed by Schmitz and Otto (1984). However they ignored the ordinal nature of the considered response time series. As an example for such a single patient analysis we investigate data collected by a 35 year old woman with chronic migraine who recorded her migraine severity four times a day on a scale from 0 to 5. To determine which weather conditions have an important effect on the migraine severity we used a proportional odds model commonly used for regression models with independent ordinal responses as a starting model for our AOP analysis. We will show that for this data the first order autocorrelation in the latent time series is high within the AOP model $(\approx 0.8)$, demonstrating considerable dependence among the measurements. We like to mention that for this patient headache severity scores are not reached by first 
successively experiencing the lowest severity category to the next higher category until the highest is reached. Therefore a continuation ratio formulation (Agresti 2002) is not appropriate for this patient. A Bayesian analysis of a probit continuation ratio formulation is given by Dunson et al. (2003) in a joint model for cluster size and sub-unit specific outcomes.

The paper is organized as follows. In Section 2 we review the proportional odds model to motivate the AOP formulation. We address the problem of variable selection and model comparison. In Section 3 we describe the data in more detail and present some results from an exploratory analysis yielding three mean specifications for the proportional odds model and two for the AOP model. In Section 4 we give the results of the model fitting and model comparison, demonstrating the superiority of the AOP model. Finally Section 5 gives a summary and draws conclusions.

\section{Models, Predictions and Model Selection}

\subsection{Models and Estimation}

In the migraine data we model an ordinal valued time series $\left\{Y_{t}, t=1, \ldots, T\right\}$, where $Y_{t} \in\{0, \ldots, K\}$ denotes the pain severity at time $t$ with ordinal levels given by $\{0, \ldots, K\}$. Together with the response $Y_{t}$ we observe further a vector $\boldsymbol{x}_{t}$ of real-valued covariates for each $t \in\{1, \ldots, T\}$ representing meteorological and time measurement information.

\subsubsection{Proportional Odds Model}

A common ordinal regression model for independent responses is the ordinal logistic model first described by Walker and Duncan (1967) and later named proportional odds model by McCullagh (1980). To aid us with the identification of important covariates in the migraine headache data we utilize the proportional odds model. The identified covariate structure will then be used in the autoregressive ordinal probit (AOP) model, which in contrast to the proportional odds model does not ignore the dependence among the measurements. The primary focus of this paper is the AOP model for which maximum likelihood estimation is not feasible and for which a Bayesian estimation approach is therefore followed. We now shortly review the proportional odds model from a threshold perspective, which motivates the AOP model formulation. For this we assume that the covariate vector $\boldsymbol{x}_{t}=\left(x_{t 1}, \ldots, x_{t p}\right)^{\prime}$ is $p$-dimensional. To model the $K+1$ different categories, an underlying unobserved real-valued time series $\left\{Y_{t}^{*}, t=1, \ldots, T\right\}$ is used which produces the discrete valued $Y_{t}$ by thresholding. In particular,

$$
\begin{aligned}
Y_{t}=k \Longleftrightarrow \quad Y_{t}^{*} \in\left(\alpha_{k-1}, \alpha_{k}\right], & k=0, \ldots, K, \\
Y_{t}^{*}=-\boldsymbol{x}_{t}^{\prime} \boldsymbol{\beta}+\varepsilon_{t}^{*}, & t=1, \ldots, T,
\end{aligned}
$$


where $-\infty=: \alpha_{-1}<\alpha_{0}<\alpha_{1}<\cdots<\alpha_{K}:=\infty$ are unknown cutpoints, and $\boldsymbol{\beta}=$ $\left(\beta_{1}, \ldots, \beta_{p}\right)^{\prime}$ is a vector of unknown regression coefficients. The errors $\varepsilon_{t}^{*}$ are assumed to be i.i.d. and follow a logistic distribution with distribution function $F(x)=\frac{\exp (x)}{1+\exp (x)}$. It is easy to see that (2.1)-(2.2) imply the more familiar representation given by

$$
P\left(Y_{t} \leq k \mid \boldsymbol{x}_{t}\right)=F\left(\alpha_{k}+\boldsymbol{x}_{t}^{\prime} \boldsymbol{\beta}\right)=\frac{\exp \left(\alpha_{k}+\boldsymbol{x}_{t}^{\prime} \boldsymbol{\beta}\right)}{1+\exp \left(\alpha_{k}+\boldsymbol{x}_{t}^{\prime} \boldsymbol{\beta}\right)}
$$

for $k=0,1, \ldots, K-1$. The properties of the proportional odds model are for example discussed in Harrell (2001) and Agresti (2002). Let $\boldsymbol{y}:=\left(y_{1}, \ldots, y_{T}\right)^{\prime}$ be the observed responses and $\boldsymbol{\alpha}:=\left(\alpha_{0}, \ldots, \alpha_{K-1}\right)^{\prime}$. Since the responses are assumed to be independent the joint likelihood is given by

$$
L(\boldsymbol{\beta}, \boldsymbol{\alpha}):=L\left(\boldsymbol{\beta}, \boldsymbol{\alpha} \mid y_{1}, \ldots, y_{T}\right)=\prod_{t=1}^{T} \pi_{t, y_{t}},
$$

where $\pi_{t k}:=P\left(Y_{t}=k \mid \boldsymbol{x}_{t}\right)=F\left(\alpha_{k}+\boldsymbol{x}_{t}^{\prime} \boldsymbol{\beta}\right)-F\left(\alpha_{k-1}+\boldsymbol{x}_{t}^{\prime} \boldsymbol{\beta}\right)$ for $k=0, \ldots, K-1$ and $\pi_{t K}:=1-\sum_{k=0}^{K-1} \pi_{t k}$. The unknown $\boldsymbol{\beta}$ and $\boldsymbol{\alpha}$ together with the ordering constraint $-\infty=: \alpha_{-1}<\alpha_{0}<\alpha_{1}<\cdots<\alpha_{K}:=\infty$ can be estimated by maximum likelihood (ML) using the S-Plus Design Library by Frank Harrell.

\subsubsection{Autoregressive Ordered Probit (AOP) Model}

Since the migraine severity at time $t$ may depend not only on the covariates at time $t$, but also on the migraine severity at time $t-1$, it may be adequate to use the autoregressive ordered probit (AOP) model introduced by Müller and Czado (2005). Here, the latent process of the common ordered probit model is extended by an autoregressive component:

$$
\begin{array}{rlrl}
Y_{t}=k \Longleftrightarrow Y_{t}^{*} \in\left(\alpha_{k-1}, \alpha_{k}\right], & k=0, \ldots, K, \\
& Y_{t}^{*}=\boldsymbol{x}_{t}^{\prime} \boldsymbol{\beta}+\phi Y_{t-1}^{*}+\varepsilon_{t}^{*}, & t & =1, \ldots, T,
\end{array}
$$

where $-\infty=: \alpha_{-1}<\alpha_{0}<\alpha_{1}<\cdots<\alpha_{K}:=\infty, \varepsilon_{t}^{*} \sim N\left(0, \delta^{2}\right)$ i.i.d., and $\boldsymbol{x}_{t}=$ $\left(1, x_{t 1}, \ldots, x_{t p}\right)^{\prime}$ is a $p+1$-dimensional vector of real-valued covariates. Accordingly, $\beta_{0}$ is the intercept for the latent process. For reasons of identifiability the cutpoint $\alpha_{0}$ is fixed to 0 , and the variance $\delta^{2}$ to 1 . For notational convenience we use again $\boldsymbol{y}:=\left(y_{1}, \ldots, y_{T}\right)^{\prime}$ for the observations and $\boldsymbol{\alpha}:=\left(\alpha_{1}, \ldots, \alpha_{K-1}\right)^{\prime}$ as for the proportional odds model, however, since $\alpha_{0}$ is fixed here, the vector $\boldsymbol{\alpha}$ has only $K-1$ components in the AOP case.

We emphasize that the right-hand side of Equation (2.2) includes the term $-\boldsymbol{x}_{t}^{\prime} \boldsymbol{\beta}$ whereas the right-hand side of Equation (2.6) uses the term $\boldsymbol{x}_{t}^{\prime} \boldsymbol{\beta}$. To make the parameters $\beta_{j}$ in model specifications (2.2) and (2.6) comparable we decided to compute the posterior mean estimates in the AOP model for the response $Y_{t}^{\circ}:=5-Y_{t}$. Therefore the worst migraine severity is associated with category 0 , and no migraine is associated with category 5 when we fit the AOP model. Hence now in both the proportional odds and in the AOP model a negative value for $\beta_{j}$ means that an increasing value of the covariate $x_{j}$ leads to a more severe migraine. 


\subsubsection{Estimation of the AOP model by GM-MGMC}

In Müller and Czado (2005) it is shown that a standard Gibbs sampling approach is extremely inefficient and cannot be recommended in practice. This inefficiency of the Gibbs sampler was already noted by Albert and Chib (1993) for polychotomous regression models and Chen and Dey (2000) for correlated ordinal regression data using lagged covariates to account for correlation. Nandram and Chen (1996) proposed a scale reparametrization for ordinal regression models with three categories, which accelerated the Gibbs sampler in this situation sufficiently. The reason for the inefficiency in ordinal response models is that the updating scheme for the cutpoints $\boldsymbol{\alpha}$ allows only small movements from one iteration to the next in larger data sets. To overcome this inefficiency Müller and Czado (2005) developed a specific grouped move multigrid Monte Carlo (GM-MGMC) Gibbs sampler for the AOP model with arbitrary number of categories. GM-MGMC Gibbs samplers have been suggested by Liu and Sabatti (2000) as a general approach to accelerate Gibbs sampling schemes.

Before we recall some more details on the GM-MGMC algorithm, we specifty prior distributions for $\boldsymbol{\alpha}, \boldsymbol{\beta}, \phi$ and $Y_{0}^{*}$. For notational convenience we define $\boldsymbol{\theta}:=\left(\boldsymbol{\beta}^{\prime}, \phi\right)^{\prime}$ and write $\boldsymbol{\alpha}_{-k}:=\left(\alpha_{1}, \ldots, \alpha_{k-1}, \alpha_{k+1}, \ldots, \alpha_{K-1}\right)^{\prime}$. Furthermore, we write $N_{I}\left(\mu, c^{2}\right)$ for a normal distribution, truncated to the interval $I$, with mean $\mu$ and variance $c^{2}$. All parameters are assumed to be a priori independent, except of the vector $\boldsymbol{\alpha}$ for which an order condition has to be satisfied. For $Y_{0}^{*}, \boldsymbol{\beta}$ and $\phi$, respectively, normal priors are chosen, and for the cutpoints a noninformative prior on the set $\left\{0<\alpha_{1}<\cdots<\alpha_{K-1}<C\right\}$, so that in total we have

$$
\pi\left(Y_{0}^{*}, \boldsymbol{\theta}, \boldsymbol{\alpha}\right) \propto \exp \left\{-\frac{1}{2}\left[\sigma^{-2}\left(Y_{0}^{*}\right)^{2}+\tau^{-2} \boldsymbol{\beta}^{\prime} \boldsymbol{\beta}+\rho^{-2} \phi^{2}\right]\right\} \cdot \mathbb{1}_{\left\{0<\alpha_{1}<\cdots<\alpha_{K-1}<C\right\}}
$$

where $\sigma, \tau, \rho$ and $C$ are known hyperparameters. One can take large values for $\sigma, \tau$ and $\rho$, when there is little prior information about $Y_{0}^{*}$ and $\boldsymbol{\theta}$. For notational convenience we redefine $\alpha_{K}:=C$, and write in the following $\boldsymbol{Y}:=\left(Y_{1}, \ldots, Y_{T}\right)^{\prime}, \boldsymbol{Y}^{*}:=\left(Y_{0}^{*}, \ldots, Y_{T}^{*}\right)^{\prime}$, and $\boldsymbol{Y}_{-t}^{*}:=\left(Y_{0}^{*}, \ldots, Y_{t-1}^{*}, Y_{t+1}^{*}, \ldots, Y_{T}^{*}\right)^{\prime}$ for $t=0, \ldots, T$.

One iteration of the GM-MGMC sampler consists of a regular iteration of the standard Gibbs sampler, which is followed by the so-called grouped move (GM) step. Hence, one complete iteration of the GM-MGMC sampler looks as follows:

\section{Latent variable update:}

Taking into account its $N\left(0, \sigma^{2}\right)$-prior, the full conditional for $Y_{0}^{*}$ is

$$
f\left(Y_{0}^{*} \mid \boldsymbol{Y}, \boldsymbol{Y}_{-0}^{*}, \boldsymbol{\alpha}, \boldsymbol{\theta}\right) \sim N\left(\frac{\phi\left(Y_{1}^{*}-\boldsymbol{x}_{1}^{\prime} \boldsymbol{\beta}\right)}{\phi^{2}+\sigma^{-2}}, \frac{1}{\phi^{2}+\sigma^{-2}}\right) .
$$

The full conditionals $f\left(Y_{t}^{*} \mid \boldsymbol{Y}, \boldsymbol{Y}_{-t}^{*}, \boldsymbol{\beta}, \phi, \boldsymbol{\alpha}\right), t=1, \ldots, T-1$ are truncated normal distributions,

$$
f\left(Y_{t}^{*} \mid \boldsymbol{Y}, \boldsymbol{Y}_{-t}^{*}, \boldsymbol{\beta}, \phi, \boldsymbol{\alpha}\right) \sim N_{\left[\alpha_{Y_{t}-1}, \alpha_{Y_{t}}\right)}\left(\frac{\phi\left(Y_{t+1}^{*}-\boldsymbol{x}_{t+1}^{\prime} \boldsymbol{\beta}\right)+\left(\boldsymbol{x}_{t}^{\prime} \boldsymbol{\beta}+\phi Y_{t-1}^{*}\right)}{1+\phi^{2}}, \frac{1}{1+\phi^{2}}\right),
$$


and for $t=T$ we have $f\left(Y_{T}^{*} \mid \boldsymbol{Y}, \boldsymbol{Y}_{-T}^{*}, \boldsymbol{\beta}, \phi, \boldsymbol{\alpha}\right) \sim N_{\left[\alpha_{Y_{T}-1}, \alpha_{Y_{T}}\right)}\left(\boldsymbol{x}_{T}^{\prime} \boldsymbol{\beta}+\phi Y_{T-1}^{*}, 1\right)$.

Joint regression and autoregressive parameter update:

Aiming in a block update of the parameters $\beta_{j}, j=0, \ldots, p$, and $\phi$, the full conditional is a $(p+2)$-dimensional normal distribution: $f\left(\boldsymbol{\theta} \mid \boldsymbol{Y}, \boldsymbol{Y}^{*}, \boldsymbol{\alpha}\right) \sim N_{p+2}\left(\Sigma Z^{\prime} \boldsymbol{Y}_{-0}^{*}, \Sigma\right)$, where the covariance matrix is given by $\Sigma:=\left(Z^{\prime} Z+\operatorname{diag}\left(\tau^{-2}, \ldots, \tau^{-2}, \rho^{-2}\right)\right)^{-1}$ and $Z$ is the $T \times(p+2)$-matrix $Z:=\left(z_{1}, \ldots, z_{T}\right)^{\prime}, z_{i}:=\left(1, x_{i 1}, \ldots, x_{i p}, Y_{i-1}^{*}\right)^{\prime}$.

\section{Cutpoint parameter update:}

For each $k=1, \ldots, K-1$, the full conditional $f\left(\alpha_{k} \mid \boldsymbol{Y}, \boldsymbol{Y}^{*}, \boldsymbol{\beta}, \phi, \boldsymbol{\alpha}_{-k}\right)$ is a uniform distribution in the interval $\left(l_{k}, r_{k}\right)$, where $l_{k}=\max \left\{\alpha_{k-1}, \max _{t=1, \ldots, T}\left\{Y_{t}^{*} \mid Y_{t}=k\right\}\right\}$ and $r_{k}=\min \left\{\alpha_{k+1}, \min _{t=1, \ldots, T}\left\{Y_{t}^{*} \mid Y_{t}=k+1\right\}\right\}$.

\section{GM step:}

The GM step transforms the current samples

$$
\boldsymbol{w}:=\left(Y_{0}^{*}, \ldots, Y_{T}^{*}, \beta_{0}, \ldots, \beta_{p}, \alpha_{1}, \ldots, \alpha_{K-1}, \phi\right)
$$

from the standard Gibbs iteration according to a randomly drawn transformation $\gamma$ from a carefully specified transformation group. For the AOP model, it turned out that the partial scale group,

$$
\Gamma_{T+p+K+1}:=\left\{\gamma>0: \boldsymbol{w} \mapsto\left(\gamma Y_{0}^{*}, \ldots, \gamma Y_{T}^{*}, \gamma \beta_{0}, \ldots, \gamma \beta_{p}, \gamma \alpha_{1}, \ldots, \gamma \alpha_{K-1}, \phi\right)\right\}
$$

accelerates the convergence of the standard Gibbs samples dramatically. Obviously, the elements $\gamma$ of this partial scale group transform all parameters and latent variables except of $\phi$ by multiplication by $\gamma$. Müller and Czado (2005) have shown that in each grouped move step (which means once in each iteration of the MCMC algorithm) the squared transformation $\gamma^{2}$ has to be drawn from a $\Gamma(a, b)$ distribution (with density $f_{a, b}(x)=b^{a} x^{a-1} e^{-b x} / \Gamma(a)$ for $x \geq 0$ ), where

$$
a=\frac{T+K+p+2}{2}, \quad b=\frac{\sum_{t=1}^{T}\left(Y_{t}^{*}-\boldsymbol{x}_{t}^{\prime} \boldsymbol{\beta}-\phi Y_{t-1}^{*}\right)^{2}+\sigma^{-2}\left(Y_{0}^{*}\right)^{2}+\tau^{-2} \boldsymbol{\beta}^{\prime} \boldsymbol{\beta}}{2} .
$$

After drawing $\gamma^{2}$ from this distribution, all parameters and latent variables except of $\phi$ have to be multiplied by $\gamma=\sqrt{\gamma^{2}}$.

\subsection{Model Selection with the Deviance Criteria}

\subsubsection{Residual Deviance Test for the Proportional Odds Model}

Here we use the deviance statistic $D$ defined as

$$
D:=2 \log \frac{\sup _{\boldsymbol{\beta}, \boldsymbol{\alpha}} L(\boldsymbol{\beta}, \boldsymbol{\alpha})}{\sup _{\boldsymbol{p}_{1}, \ldots, p_{T}} L\left(\boldsymbol{p}_{1}, \ldots, \boldsymbol{p}_{T}\right)}
$$


where $L(\boldsymbol{\beta}, \boldsymbol{\alpha})$ is defined in (2.4) and the supremum is taken over all $\boldsymbol{\alpha}$ which satisfy the ordering constraint. Further we denote by $L\left(\boldsymbol{p}_{1}, \ldots, \boldsymbol{p}_{T}\right)$ for $\boldsymbol{p}_{t}:=\left(p_{t 0}, \ldots, p_{t k}\right)^{\prime}$ the joint likelihood of $\mathrm{T}$ independent discrete random variables $Z_{t}$ taking on values $0, \ldots, K$ with probabilities $p_{t 0}, \ldots, p_{t K}$, respectively. We call $L\left(\boldsymbol{p}_{1}, \ldots, \boldsymbol{p}_{T}\right)$ the likelihood of the corresponding unstructured model. It is straight forward to show that $D:=\sum_{t=1}^{T} \sum_{k=1}^{K} \log \left(\bar{\pi}_{t k}\right)$, where $\bar{\pi}_{t k}:=F\left(\bar{\alpha}_{k}+\boldsymbol{x}_{t}^{\prime} \overline{\boldsymbol{\beta}}\right)-F\left(\bar{\alpha}_{k-1}+\boldsymbol{x}_{t}^{\prime} \overline{\boldsymbol{\beta}}\right)$ and $\overline{\boldsymbol{\beta}}$ and $\overline{\boldsymbol{\alpha}}$ the joint MLE of $\boldsymbol{\beta}$ and $\boldsymbol{\alpha}$ under the ordering constraint for $\alpha$. Note that the proportional odds model can be considered as a special case of multi categorical models considered in Tutz (2000). Here he shows that the null hypothesis of model adequacy can be rejected at level $\alpha$ if $D>\chi_{T \cdot K-p, 1-\alpha}^{2}$, where $T$ is the number of observations, $K$ the number of categories minus one and $p$ the number of regression parameters to be estimated. The $\chi^{2}$ approximation is most accurate when covariates are categorical and the expected cell counts formed by the cross classification of the responses and covariates are greater than 5. Alternative goodness-of-fit tests in ordinal regression models have been suggested in Lipsitz et al. (1996). We restrict our attention to the residual deviance, since we want to use the deviance information criterion for the AOP model, which is closely related to the deviance.

\subsubsection{Deviance Information Criterion for the AOP model}

The Deviance Information Criterion (DIC) was suggested as general model selection criterion by Spiegelhalter et al. (2002). Model fit is measured by the Bayesian deviance defined as $D(\boldsymbol{\theta}):=-2 \log \{f(\boldsymbol{y} \mid \boldsymbol{\theta})\}+2 \log \{f(\boldsymbol{y})\}$. The standardizing term $2 \log \{f(\boldsymbol{y})\}$ for the AOP model will be set to zero, which is consistent with a unstructured model. Model complexity is measured by the effective number of model parameters defined as $p_{D}:=\overline{D(\boldsymbol{\theta})}-D(\overline{\boldsymbol{\theta}})$, where $\overline{D(\boldsymbol{\theta})}:=E(D(\boldsymbol{\theta}) \mid \boldsymbol{y})$ and $D(\overline{\boldsymbol{\theta}})=D(E(\boldsymbol{\theta} \mid \boldsymbol{y}))$. Spiegelhalter et al. (2002) suggest to use

$$
\mathrm{DIC}:=D(\overline{\boldsymbol{\theta}})+2 p_{D}=\overline{D(\boldsymbol{\theta})}+p_{D}=2 \overline{D(\boldsymbol{\theta})}-D(\overline{\boldsymbol{\theta}}) .
$$

as model selection criterion. A model with smaller DIC is preferred. We note that the DIC allows for an information theoretic interpretation in exponential family models (van der Linde 2005) and might be less reliable in non exponential family models such as the AOP model.

For the AOP model the parameter $\boldsymbol{\theta}$ includes the cutpoint vector $\boldsymbol{\alpha}$, the regression parameter vector $\boldsymbol{\beta}$, the autoregressive parameter $\phi$, and all the latent variables $Y_{t}^{*}$. The Bayesian deviance for the model is

$$
\begin{aligned}
D(\theta) & =-2 \log f(\boldsymbol{y} \mid \boldsymbol{\theta}) \\
& =-2 \sum_{t=1}^{T} \log \left[\Phi\left(\alpha_{k}-\boldsymbol{x}_{t}^{\prime} \boldsymbol{\beta}-\phi Y_{t-1}^{*}\right)-\Phi\left(\alpha_{k-1}-\boldsymbol{x}_{t}^{\prime} \boldsymbol{\beta}-\phi Y_{t-1}^{*}\right)\right] .
\end{aligned}
$$

To compute the DIC, the expression $\overline{D(\boldsymbol{\theta})}$ can be estimated by averaging the terms $D\left(\boldsymbol{\theta}_{i}\right)$, where $\boldsymbol{\theta}_{i}$ denotes the random sample for $\boldsymbol{\theta}$ drawn in iteration $i$ of the MCMC sampler. The 
value of $D(\overline{\boldsymbol{\theta}})$ is given by inserting the corresponding posterior mean estimates in Equation (2.7).

We mention that the DIC as defined above considers the latent variables as the focus of the analysis. However in our application this is not the case. It would be more appropriate to consider a DIC measure based on the marginalized likelihood, i.e. the likelihood where the latent variables are integrated out. However this would involve a very high dimensional integration (in our application this dimension would be 1072), which is even numerically intractable.

\subsection{Bayes Factors}

Since DIC might be unreliable for the AOP model we consider Bayes factors based on the marginal likelihood as an alternative method for model comparison (see Kass and Raftery (1995)). Müller and Czado (2005) provided an estimation procedure for the marginal likelihood for the AOP model adapting the methods of Chib (1995) and Chib and Jeliazkov (2001). In particular, the Bayes factor of a model $M_{1}$ versus a model $M_{2}$ is given by

$$
B\left(\boldsymbol{y} \mid M_{1}, M_{2}\right):=\frac{m\left(\boldsymbol{y} \mid M_{1}\right)}{m\left(\boldsymbol{y} \mid M_{2}\right)},
$$

where $m(\boldsymbol{y} \mid M):=\int f(\boldsymbol{y} \mid \boldsymbol{\theta}, M) p(\boldsymbol{\theta} \mid M) d \boldsymbol{\theta}$ is the marginal likelihood of model $M$. Here $p(\boldsymbol{\theta} \mid M)$ and $f(\boldsymbol{y} \mid \boldsymbol{\theta}, M)$ denote the prior of the parameters $\boldsymbol{\theta}$ and the likelihood in model $M$, respectively. Using the definition of the posterior distribution $p(\boldsymbol{\theta} \mid \boldsymbol{y}, M)$ in Model $\mathbf{M}$ the marginal likelihood of Model M can be estimated by

$$
m(\boldsymbol{y} \mid M)=\frac{f\left(\boldsymbol{y} \mid \boldsymbol{\theta}^{o}, M\right) p\left(\boldsymbol{\theta}^{o} \mid M\right)}{p\left(\boldsymbol{\theta}^{o} \mid \boldsymbol{y}, M\right)}
$$

for every value of $\boldsymbol{\theta}^{\circ}$. For reasons of efficiency we set $\boldsymbol{\theta}^{\circ}$ equal to the posterior mean estimate of $\boldsymbol{\theta}$. Whereas $p\left(\boldsymbol{\theta}^{o} \mid M\right)$ can be computed exactly, the other two factors on the right hand side of (2.8) have to be estimated. To this end one uses a particle filter and the output from reduced runs of the MCMC estimation procedure. For the AOP models the model parameters are given by the cutpoints, the regression parameters and the autoregressive parameter.

\subsection{Pseudo-predictions}

One intuitive and quite simple way to investigate the quality of a model fit is to compute pseudo-predictions. In the proportional odds model this means that one predicts the response at time $t$ using ML estimates for the regression parameters and cutpoints which are plugged into the model equations. This results in a forecast probability for each category. One can use the category with highest forecast probability as prediction for the response at 
time $t$. However, when the ML estimates are based on the whole data set we call these predictions more precisely pseudo-predictions. For the AOP model one uses posterior mean estimates instead of the ML estimates. Here, of course, one also needs a posterior mean estimate of $Y_{t-1}^{*}$.

\subsubsection{Pseudo-predictions for the Proportional Odds Model}

The fitted probabilities for the proportional odds model for each category at time $t$ are defined by

$$
\begin{aligned}
& \bar{\pi}_{t 0}:=\hat{P}\left(Y_{t}=0 \mid \boldsymbol{x}_{t}, \overline{\boldsymbol{\alpha}}, \overline{\boldsymbol{\beta}}\right)=\frac{\exp \left(\bar{\alpha}_{0}+\boldsymbol{x}_{t}^{\prime} \overline{\boldsymbol{\beta}}\right)}{1+\exp \left(\bar{\alpha}_{0}+\boldsymbol{x}_{t}^{\prime} \overline{\boldsymbol{\beta}}\right)}, \\
& \bar{\pi}_{t k}:=\hat{P}\left(Y_{t}=k \mid \boldsymbol{x}_{t}, \overline{\boldsymbol{\alpha}}, \overline{\boldsymbol{\beta}}\right)=\frac{\exp \left(\bar{\alpha}_{k}+\boldsymbol{x}_{t}^{\prime} \overline{\boldsymbol{\beta}}\right)}{1+\exp \left(\bar{\alpha}_{k}+\boldsymbol{x}_{t}^{\prime} \overline{\boldsymbol{\beta}}\right)}-\frac{\exp \left(\bar{\alpha}_{k-1}+\boldsymbol{x}_{t}^{\prime} \overline{\boldsymbol{\beta}}\right)}{1+\exp \left(\bar{\alpha}_{k-1}+\boldsymbol{x}_{t}^{\prime} \overline{\boldsymbol{\beta}}\right)}, \\
& \bar{\pi}_{t K}:=1, \ldots, K-1, \\
& \quad:=\hat{P}\left(Y_{t}=K \mid \boldsymbol{x}_{t}, \overline{\boldsymbol{\alpha}}, \overline{\boldsymbol{\beta}}\right)=1-\frac{\exp \left(\bar{\alpha}_{K-1}+\boldsymbol{x}_{t}^{\prime} \overline{\boldsymbol{\beta}}\right)}{1+\exp \left(\bar{\alpha}_{K-1}+\boldsymbol{x}_{t}^{\prime} \overline{\boldsymbol{\beta}}\right)}
\end{aligned}
$$

where $\overline{\boldsymbol{\alpha}}$ and $\overline{\boldsymbol{\beta}}$ denote maximum likelihood estimates of $\boldsymbol{\alpha}$ and $\boldsymbol{\beta}$, respectively. The corresponding pseudo-prediction of $Y_{t}$ is therefore given by the category $k$, which has the highest value among $\bar{\pi}_{t 0}, \ldots, \bar{\pi}_{t K}$.

\subsubsection{Pseudo-predictions for the AOP model}

The corresponding posterior probability estimates in the AOP model for each category at time $t$ are defined by

$$
\begin{aligned}
\bar{\pi}_{t 0} & :=\hat{P}\left(Y_{t}=0 \mid \boldsymbol{x}_{t}, \overline{\boldsymbol{\alpha}}, \overline{\boldsymbol{\beta}}, \bar{\phi}, \bar{Y}_{t-1}^{*}\right)=\Phi\left(\bar{\alpha}_{0}-\boldsymbol{x}_{t}^{\prime} \overline{\boldsymbol{\beta}}-\bar{\phi} \bar{Y}_{t-1}^{*}\right) \\
\bar{\pi}_{t k} & :=\hat{P}\left(Y_{t}=k \mid \boldsymbol{x}_{t}, \overline{\boldsymbol{\alpha}}, \overline{\boldsymbol{\beta}}, \bar{\phi}, \bar{Y}_{t-1}^{*}\right)=\Phi\left(\bar{\alpha}_{k}-\boldsymbol{x}_{t}^{\prime} \overline{\boldsymbol{\beta}}-\bar{\phi} \bar{Y}_{t-1}^{*}\right) \\
& -\Phi\left(\bar{\alpha}_{k-1}-\boldsymbol{x}_{t}^{\prime} \overline{\boldsymbol{\beta} \phi} \bar{Y}_{t-1}^{*}\right), \quad k=1, \ldots, K-1, \\
\bar{\pi}_{t K} & :=\hat{P}\left(Y_{t}=K \mid \boldsymbol{x}_{t}, \overline{\boldsymbol{\alpha}}, \overline{\boldsymbol{\beta}}, \bar{\phi}, \bar{Y}_{t-1}^{*}\right)=1-\Phi\left(\bar{\alpha}_{K-1}-\boldsymbol{x}_{t}^{\prime} \overline{\boldsymbol{\beta}}-\bar{\phi} \bar{Y}_{t-1}^{*}\right) .
\end{aligned}
$$

where $\overline{\boldsymbol{\alpha}}, \overline{\boldsymbol{\beta}}, \bar{\phi}$, and $\bar{Y}_{t-1}^{*}$ denote posterior mean estimates of the corresponding parameters and latent variables. The corresponding pseudo-prediction of $Y_{t}$ are therefore given by the category $\mathrm{k}$ which has the highest value among $\bar{\pi}_{t 0}, \ldots, \bar{\pi}_{t K}$. 


\subsubsection{Assessing Model fit based on Pseudo-predictions}

Now we suggest to use the pseudo-predictions for model assessment. For this we define the variables $P_{t k}^{o b s}$ which correspond to the 'observed' probabilities for category $k$ at time $t$ in contrast to the 'predicted' probabilities $\bar{\pi}_{t k}$ defined in the previous subsections:

$$
P_{t k}^{o b s}:= \begin{cases}1 & \text { if } Y_{t}=k \\ 0 & \text { else. }\end{cases}
$$

When category $k$ is observed at time $t$, it is clear that a good model fit leads to a high probability $\bar{\pi}_{t k}$, and to small probabilities $\bar{\pi}_{t j}$ for the other categories $j \neq k$. A large difference should be punished more than a small difference. Therefore we compute the verification score introduced by Brier (1950) defined by

$$
\mathrm{S}:=\frac{1}{T} \sum_{k=0}^{K} \sum_{t=1}^{T}\left(P_{t k}^{o b s}-\bar{\pi}_{t k}\right)^{2}
$$

to get an idea of the model fit. Of course, the smaller the value of S, the better the model. The Brier score has been heavily used to evaluate forecasts in the meteorological sciences and has the attractive property of being a strictly proper scoring rule (see for example Gneiting and Raftery (2004)).

\section{Analysis of migraine severity data}

\subsection{Data description and exploratory analysis}

We investigate the migraine headache diary of a 35 year old female, who is working fulltime as a manager. She suffers from migraine without aura for 22 years. In this study she recorded her headache four times a day on an ordinal scale from 0 to 5 , where 0 means that she did not feel any migraine headache, and 5 the worst migraine headache she can feel. For a precise definition of the migraine intensity categories see Table 1 . The data is part of a larger study on determinants of migraine headaches collected by the psychologist T. Kostecki-Dillon, York University, Toronto, Canada. The migraine headache diary was completed between January 6, 1995, and September 30, 1995, which is a period of 268 subsequent days. Therefore the length of the data set is $4 \cdot 268=1072$. In addition also weather related information on a daily basis was collected. This includes information on humidity, windchill, temperature and pressure changes, wind direction, and length of sun shine on the previous day.

Table 1 contains also the frequencies for the six possible response categories in the data set. As can be seen from this table 150 observations are unequal to zero which corresponds to suffering from migraine headaches in about $14 \%$ of the time. On the one hand we use covariates which reflect weather conditions, on the other hand covariates which contain 
information about the measurement time points. A description of the covariates in our analysis is also provided in Table 1 . We point out that the humidity index is measured only in the period from May to October and the windchill index only in the period from November to April. This means that always only one of these covariates is contained in the data set.

[Table 1 about here]

In the following we conduct a short exploratory analysis. As described in Müller and Czado (2005), the idea is to compute the average response for each category of a categorical covariate and for intervals, when a continuous covariate is considered. More precisely for a continuous covariate $x_{t j}$ which falls in an interval $I$ with $n_{I}$ observations, the average response is given as

$$
\bar{Y}_{I}\left(x_{\cdot j}\right):=\frac{1}{n_{I}} \sum_{t: x_{t j} \in I} Y_{t}
$$

Depending on the shape of the graph one can then decide to use an appropriate transformation of the covariate or to use indicator variables, which is, of course, the most flexible way of modeling.

PMND1P (mean pressure change from previous day, cf. Figure 1, top panel): We group the observed PMND1P values into six intervals with equal number of observations and compute the average response for each interval. A linear relationship seems to be sufficient, since a possibly present quadratic part is obviously small.

[Figure 1 about here]

S1P (sunshine on previous day): This covariate has not been collected during all 30 days of April 1995, so that for 120 observations this covariate is missing in the period under consideration. The remaining 952 observations are grouped in intervals. The relationship is quite linear (not shown), and a sunny day seems to increase the probability for headache on the following day, since the average response increases with the length of the sunshine. The range of the average response is 0.31 .

HDXDD (humidity index): We computed the average response for each interval and decided to use a quadratic transformation. The relative high range among these average responses of 0.83 is a first hint at the importance of this covariate.

WCD (windchill): We use an indicator for windchill. If windchill is present, the patient suffered from more intense migraine headaches.

WDAY (weekday): Because of the periodicity a polynomial or logarithmic transformation does not make sense. Perhaps a sine transformation could be used. We use indicator variables since this choice provides the most flexible way for modeling the influence of the weekdays. Weekdays were grouped together when they showed a similar behavior. 
Indicator variables are abbreviated in a natural way. For example, the variable TUEWED is 1 if the measurement was done on a Tuesday or Wednesday, otherwise 0 .

MESS (time of measurement, cf. Figure 1, bottom panel): In the afternoon the average response is the highest with 0.51 . The difference between the range of the average response is $0.51-0.26=0.25$. The afternoon indicator HAMP.IND is used.

\subsection{Proportional Odds Model Specifications}

To determine reasonable mean specifications for the AOP model we ignore in an initial analysis the dependence among the responses and utilize the proportional odds model. For the proportional odds model we analyzed models with different sets of covariates. As mentioned above, the covariate 'sunshine on previous day' has not been collected during April 1995. This covariate, however, seems to be important for the analysis, so we decided to remove all observations from April 1995 and to reduce our data set to the length $1072-$ $120=952$. Since the proportional odds model does not take any autoregressive dependence between the observations into account, no selection bias occurs. The three models A, B, and $\mathrm{C}$ considered in the following are found by a forward selection procedure. In each step the $p$-values for each covariate were determined by a Wald test. The covariate with the lowest $p$-value below the $5 \%$ level was included. The $p$-values of already included variables were checked that they remain below a $5 \%$ level and otherwise removed. This means that the covariates of Model A, B, and C are all significant on the $5 \%$ level.

Model A contains only main effects. For time of measurement we use only an indicator for the afternoon measurement and an indicator for Tuesday or Wednesday. In Model B and $\mathrm{C}$ we consider three weekday indicators following our exploratory analysis. Furthermore, in Model B we also allow for three interaction effects, whereas Model C contains nine interaction components. The covariates which are used are seen in Table 2. This table also gives the ML estimators for the regression coefficients and the cutpoints.

[Table 2 about here]

\subsection{AOP Model Specifications}

For the AOP model with latent variables given by

$$
Y_{t}^{*}=x_{t}^{\prime} \boldsymbol{\beta}+\phi Y_{t-1}^{*}+\varepsilon_{t}^{*}
$$

we investigate two models. For numerical stability we use covariates which have been standardized such that they have empirical mean 0 and empirical variance 1 . We call these 
standardized covariates $\boldsymbol{x}_{i}^{s}=\left(x_{1 i}^{s}, \ldots, x_{T i}^{s}\right)^{\prime}$, where the components are given by

$$
x_{t i}^{s}:=\frac{x_{t i}-\bar{x}_{. i}}{\sqrt{\frac{1}{n} \sum_{t=1}^{T}\left(x_{t i}-\bar{x}_{. i}\right)^{2}}}
$$

with $\bar{x}_{. i}=\frac{1}{n} \sum_{t=1}^{T} x_{t i}$. Only indicator variables $\boldsymbol{x}_{. i}$ (where $x_{t i} \in\{0,1\}$ for all $t \in\{1, \ldots, T\}$ ) are not standardized. The proportional odds model specifications from above were used as a starting point for the model specifications of the AOP models considered. Note that for the AOP model a selection bias (due to cutting out the observations in April 1995) might occur. Since, however, the used data set still has 952 observations and the observations which have been cut out are concentrated in one single cluster, this possibly occurring selection bias seems to be negligible. If the $95 \%$ credible interval of a parameter contained zero, the corresponding covariate was removed from the model. In this way the proportional odds models A and B lead to the AOP models I and II, respectively.

Table 3 shows the posterior mean estimates together with estimated $2.5 \%$ and $97.5 \%$ quantiles for all parameters based on 10000 iterations with a burn-in of 1000 iterations (this choice seemed to be reasonable after an inspection of the chains produced by the GMMGMC sampler; it goes along with the results of Müller and Czado (2005), where in several simulation settings a burn-in period of 1000 iterations always turned out to be sufficiently long). For Model I, the $95 \%$ credible interval for every main effect does not contain zero, so every covariate is significant. For Model II, the $95 \%$ credible intervals for $\mathrm{PMND}_{t} \mathrm{P}_{t}^{s}$ and $\mathrm{WEDFRI}_{t}$ contain the value 0 . However, these two covariates must remain in the model since they appear in an interaction term which is itself significant. According to Müller and Czado (2005), the priors of all regression coefficients were normal with mean 0 and standard deviation 10 , so that they are quite uniformative compared to the magnitude of the estimates in Table 3. The prior of $\phi$ was truncated normal with mean 0 and standard deviation 10 , therefore again quite uninformative on $(-1,1)$.

[Table 3 about here]

\section{Results}

Now we conduct a model comparison analysis for the five models investigated in Sections 3.2 and 3.3. First we consider the proportional odds models. To decide which of the proportional odds models fits the data best, we use the residual deviance test of Section 2.2.1. As mentioned there a model does not describe the data well, if $D>\chi_{T \cdot K-p, 1-\alpha}^{2}$. Here we have $T=952$ and $K=5$. We test on the $5 \%$ and $1 \%$ level and compute the $p$-value. Table 2 shows the results of the deviance analysis for the three models. For all three models the deviance $D$ is not larger than the corresponding $99 \%$ quantiles of the $\chi^{2}$-distribution, therefore all considered models fit the data quite well. Next we compare 
the AOP models using the DIC criterion. The values of the DIC for Model I and Model II are given in Table 3. The posterior mean of the Bayesian deviance $\overline{D(\boldsymbol{\theta})}$ is smaller for Model II, however the complexity measure $p_{D}$ is smaller for the more complex Model II, which indicates that DIC might not be suitable for AOP models. Therefore we prefer to base our model selection on Bayes factors and Brier scores. We see from the likelihood ordinate, that Model II clearly fits the data better than Model I (by the likelihood factor $\exp (-407.8493+417.5238)=15906.77)$. However, the prior and the posterior ordinate punish Model II heavily, since it uses four covariates more than Model I. Therefore, if one uses the Bayes factor as model selection criterion, one should prefer the simpler Model I to describe the data, since following the Bayes factor scale by Jeffreys (1961), the evidence of Model I against Model II is decisive.

Finally we compare all proportional odds models and AOP models using the pseudopredictions defined in Section 2.4. The corresponding Brier scores are given in Table 2 and 3, respectively. We conclude that the two AOP models describe the data better than all the proportional odds models. The Brier scores chooses Model I over all models, which is consistent with the model selection based on Bayes factors. Therefore we conclude that Model I is the overall preferred model for this data set.

The signs of the regression parameters in Table 3 agree nearly everywhere with the signs in Table 2. This means that both the proportional odds models and the AOP models lead to the same conclusions, when asking which covariates have a high and which a low value to reduce the migraine severity. For example from the negative signs for S1P in all models we conclude that a sunny day increases the headache severity on the next day. This agrees with our conjecture from the exploratory analysis. The indicator for afternoon, HAPM.IND, also has a coefficient with negative sign. Again this approves our conjecture: the afternoon headache is usually worse than in the morning, at noon, and during the night. Considering the coefficients of the weekday indicators in Model II we see that the headache is worse between Wednesday and Saturday which might be a consequence of an (over)exertion on the job.

We provide now a quantitative interpretation of the covariate effects in the AOP models. For this we match the first two moments of the standard normal distribution to the logistic distribution to give the approximation

$$
\Phi(z) \approx \frac{\exp \left(\frac{\pi}{\sqrt{3}} z\right)}{1+\exp \left(\frac{\pi}{\sqrt{3}} z\right)}
$$

For the AOP model it follows that the cumulative log odds ratio $l_{t}\left(k \mid \boldsymbol{x}_{t}\right)$ for category $\mathrm{k}$ at time t and covariate vector $\boldsymbol{x}_{t}$ can be approximated by

$$
l_{t}\left(k \mid \boldsymbol{x}_{t}\right):=\log \left(\frac{F_{t}\left(k \mid \boldsymbol{x}_{t}\right)}{1-F_{t}\left(k \mid \boldsymbol{x}_{t}\right)}\right) \approx \frac{\pi}{\sqrt{3}}\left(\alpha_{k}-\boldsymbol{x}_{t}^{\prime} \boldsymbol{\beta}-\phi Y_{t-1}^{*}\right)
$$

where $F_{t}\left(k \mid \boldsymbol{x}_{t}\right):=P\left(Y_{t} \leq k \mid \boldsymbol{x}_{t}, \boldsymbol{\alpha}, \boldsymbol{\beta}, \phi, Y_{t-1}^{*}\right)=\Phi\left(\alpha_{k}-\boldsymbol{x}_{t}^{\prime} \boldsymbol{\beta}-\phi Y_{t-1}^{*}\right)$. Therefore the 
scaled impact of covariate $x_{j}$ defined as

$$
\frac{\pi}{\sqrt{3}}\left(\beta_{0}+\beta_{j} x_{j}\right)
$$

approximates the effect on the cumulative log odds ratio, when the remaining covariates are set to zero. Note that $F_{t}\left(k \mid \boldsymbol{x}_{t}\right)$ corresponds to the probability of experiencing a headache of category k or worse at time $\mathrm{t}$ and covariate vector $\boldsymbol{x}_{t}$, since we use $Y_{t}^{o}=5-Y_{t}$ in the AOP models. Since we used standardized covariates a zero standardized covariate value corresponds to the average value of the unstandardized covariate. The scaled impacts of the unstandardized covariates HDXDD and S1P are given in Figure 2. Recall that HDXDD enters the model in a quadratic fashion. The corresponding $95 \%$ credible intervals show that the data provides much more evidence of a sunshine effect on the previous day than a humidity effect.

[Figure 2 about here]

Using 4.10 we can approximate the cumulative odds ratio change by

$$
\frac{\frac{F_{t}\left(k \mid \boldsymbol{x}_{1}\right)}{1-F_{t}\left(k \mid \boldsymbol{x}_{1}\right)}}{\frac{F_{t}\left(k \mid \boldsymbol{x}_{2}\right)}{1-F_{t}\left(k \mid \boldsymbol{x}_{2}\right)}} \approx \exp \left\{\frac{\pi}{\sqrt{3}}\left(\boldsymbol{x}_{2}-\boldsymbol{x}_{1}\right)^{\prime} \boldsymbol{\beta}\right\}
$$

when the covariate vector is changed from $b x_{t}=\boldsymbol{x}_{1}$ to $\boldsymbol{x}_{t}=\boldsymbol{x}_{2}$. Note that this quantity is independent of category $\mathrm{k}, \phi$ and $Y_{t-1}^{*}$. Table 4 gives these cumulative odds ratio changes when a single covariate is changed. The remaining covariate values are held fixed. We see that the presence of windchill has the largest impact on the cumulative odds ratio change followed by a PM measurement and exposure to sunshine on the previous day. The evidence for a humidity effect on the cumulative odds ratio change is marginal since the $95 \%$ credible intervals contain 1. In particular this means that the odds of having a headache of severity $\mathrm{k}$ or worse is 4.6 (2.93) times higher when windchill (PM measurement) is present compared to being absent. Five hours more sunshine on the previous day changes the cumulative odds ratio by a factor of 1.30 .

[Table 4 about here]

Finally we note that the autoregressive component for the latent time series $Y_{t}^{*}$ is around 0.8 indicating large positive dependence among the ordinal intensity measurements.

In summary we recommend to this patient to avoid windchill and long sunshine exposures. The evidence for a humidity, workday and pressure change effect is too small to warrant specific recommendations with regard to these variables. Further the chance of experiencing a headache compared to no headache is about three times higher in the afternoon compared to other times of the day. 


\section{Conclusions}

The importance of using time series models to evaluate within patient migraine headache diaries has also been recognized in a recent paper by Houle et al. (2005). As in Prince et al. (2004) they study the daily total and maximum score over four measurements of a patient over one month. They recognized that this approach yields only a time series of length 28 , which is considered too short to make significant conclusions about the time series properties. In contrast our approach uses non-aggregated data and thus longer time series. In addition we avoid information loss due to data aggregation. The analysis of Houle et al. (2005) showed the presence of positive autocorrelations between successive values of their daily outcome measures. They however do not consider time series models to account for this autocorrelation due to their short time series length. Further, they only included a linear time trend as explanatory variable for their headache outcome variable. Our approach overcomes these short comings - short time series due to data aggregation, no model based adjustment for autocorrelation and a very limited set of explanatory factors for headache activity.

For our approach we applied the autoregressive ordered probit (AOP) model suggested by Müller and Czado (2005) to an ordinal valued time series arising from headache intensity assessments. Here the ordered categories are produced by threshholding a latent real-valued time series with regression effects. To model the dependencies among the measurements the latent time series includes not only regression components but also an autoregressive component. Parameter estimation is facilitated using a grouped move multigrid Monte Carlo (GM-MGMC) Gibbs sampler in a Bayesian setting. Models were compared using Bayes factors and the Brier score based on pseudo predictions. We also show that the DIC model selection criterion is problematic for AOP models.

For the migraine headache intensity data the latent time series shows a high first order autocorrelation of around 0.8 demonstrating considerable dependence among the ordinal measurements. For this patient we were able to demonstrate considerable impact of weather related variables such as the present of windchill and sunshine length. This supports the conclusions of Prince et al. (2004) who showed that some patients are sensitive to weather. Specific recommendations to this patient to lower the risk factors for severe migraine headaches have been provided.

Even though an individual analysis offers the opportunity to develop more precise migraine control mechanisms, it is of interest to identify common risk factors in groups of patients. This problem is subject of current research. A possibility is to generalize the AOP model to a multivariate setting, with the same autoregressive structure for the latent variables. However, also other similar multivariate models could be used, for example the model discussed in Heiss (2008) (which is estimated using sequential Gaussian quadrature) or the bivariate model proposed in Todem et al. (2007) (which is estimated using adaptive Gaussian quadrature). Another possibility could be the multivariate extension of the dynamic logit model which was developed by Bartolucci and Farcomeni (2009). Due to the involved latent process, a direct maximum likelihood estimation is not possible here, and the authors 
suggested an EM algorithm to estimate the model.

\section{Acknowledgements}

This work was supported by the Deutsche Forschungsgemeinschaft, Sonderforschungsbereich 386 Statistical Analysis of Discrete Structures. We would like to thank T. KosteckiDillon for providing the data.

\section{References}

Agresti, A. (2002). Categorical Data Analysis (2 ed.). New York: John Wiley \& Sons.

Albert, J. and S. Chib (1993). Bayesian analysis of binary and polychotomous response data. Journal of the American Statistical Association 88, 669-679.

Bartolucci, F. and A. Farcomeni (2009). A multivariate extension of the dynamic logit model for longitudinal data based on a latent Markov heterogeneity structure. Journal of the American Statistical Association 104, 816-831.

Brier, G. (1950). Verification of forecasts expressed in terms of probability. Monthly Weather Review 7, 1-3.

Chen, M. and D. Dey (2000). Bayesian analysis for correlated ordinal data models. In Generalized linear Models - A Bayesian Perspective (eds Dey, D.K. and Ghosh, S.K. and Mallick, B.K. New York: Marcel Dekker.

Chib, S. (1995). Marginal likelihood from the Gibbs output. Journal of the American Statistical Association 90, 1313-1321.

Chib, S. and I. Jeliazkov (2001). Marginal likelihood from the Metropolis-hastings output. Journal of the American Statistical Association 96, 270-281.

Cooke, L., M. Rose, and W. Becker (2000). Chinook winds and migraine headache. Neurology 54(2), 302-307.

Dunson, D., Z. Chen, and J. Harry (2003). A Bayesian approach for joint modeling of cluster size and subunit-specific outcomes. biometrics 59, 521-530.

Fahrmeir, L., C. Gieger, and C. Hermann (1999). An application of isotonic longitudinal marginal regression to monitoring the healing process. Biometrics 55, 951-956.

Fahrmeir, L. and L. Pritscher (1996). Regression analysis of forest damage by marginal models for correlated ordinal responses. Journal of Environmental and Ecological Statistics 3, 257-268.

Gieger, C. (1997). Non- and semiparametric marginal regression models for ordinal response. SFB Discussion Paper 71, Institut für Statistik, Ludwig-MaximiliansUniversität, München, Germany. 
Gneiting, T. and A. Raftery (2004). Strictly proper scoring rules, prediction and estimation. Technical report No. 463, Department of Statistics, University of Washington, Seattle, U.S.A..

Harrell, F. (2001). Regression Modeling Strategies. New York: Springer Verlag.

Heagerty, P. and S. Zeger (1996). Marginal regression models for clustered ordinal measurements. Journal of the American Statistical Association 91, 809-822.

Heiss, F. (2008). Sequential numerical integration in nonlinear state space models for microeconometric panel data. Journal of Applied Econometrics 23, 373-389.

Houle, T., D. Penzien, and J. Rains (2005). Time-series features of headache: individual distributions, patterns and predictability of pain. Headache 45, 445-458.

Jeffreys, H. (1961). Theory of Probability (3 ed.). Claredon Press, Oxford.

Kass, R. and A. Raftery (1995). Bayes factors. Journal of the American Statistical Association $90,773-795$.

Kauermann, G. (2000). Modeling longitudinal data with ordinal response by varying coefficients. Biometrics 56, 692-698.

Liang, K.-Y., S. Zeger, and B. Qaqish (1992). Multivariate regression analyses for categorical data (with discussion). Journal of the Royal Statistical Society B 54, 2-24.

Lipsitz, S., G. Fitzmaurice, and G. Molenberghs (1996). Goodness-of-fit test for ordinal response regression models. Applied Statistics 45, 175-190.

Liu, J. and C. Sabatti (2000). Generalised Gibbs sampler and multigrid Monte Carlo for Bayesian computation. Biometrika 87, 353-369.

McCullagh, P. (1980). Regression models for ordinal data (with discussion). Journal of the Royal Statistical Society B 42, 109-142.

Müller, G. and C. Czado (2005). An Autoregressive Ordered Probit Model with Application to High-Frequency Finance. Journal of Computational and Graphical Statistics 14, 320-338.

Nandram, B. and M. Chen (1996). Accelerating Gibbs sampler convergence in generalized linear models via a reparametrization. Journal of Statistical Computations and Simulation 45, 129-144.

Piorecky, J., W. Becker, and M. Rose (1996). The effect of Chinook Winds on the probability of Migraine headache Occurence. Headache 37, 153-158.

Prince, P., A. Rapoport, F. Sheftell, S. Tepper, and M. Bigal (2004). The effect of weather on headache. Headache 44, 153-158.

Schmitz, B. and J. Otto (1984). Die Analyse von Migraine mit allgemeinpsychologischen Stresskonzepten. Zeitreihenanalysen für einen Einzelfall (in German). Archiv der Psychologie 136, 211-234.

Spiegelhalter, D. J., N. G. Best, B. P. Carlin, and A. van der Linde (2002). Bayesian measures of model complexity and fit. Journal of the Royal Statistical Society B 64, 583-639. 
Todem, D., K. Kim, and E. Lesaffre (2007). Latent-variable models for longitudinal data with bivariate ordinal outcomes. Statistics in Medicine 26, 1034-1054.

Tutz, G. (2000). Die Analyse Kategorialer Daten (in German). München: Oldenbourg. van der Linde, A. (2005). DIC in variable selection. Statistica Neerlandia 59, 45-56.

Walker, S. and D. Duncan (1967). Estimation of the probability of an event as a function of several independent variables. Biometrika 54, 167-178.

Wild, C. and T. Yee (1996). Additive extensions to generalized estimating equation methods. Journal of the Royal Statistical Society B 58, 711-725.

Zeger, S. and K.-Y. Liang (1986). Longitudinal data analysis for discrete and continuous outcomes. Biometrics 42, 121-130. 
Table 1: Description of response scales with observed frequencies and weather and time measurements related covariates

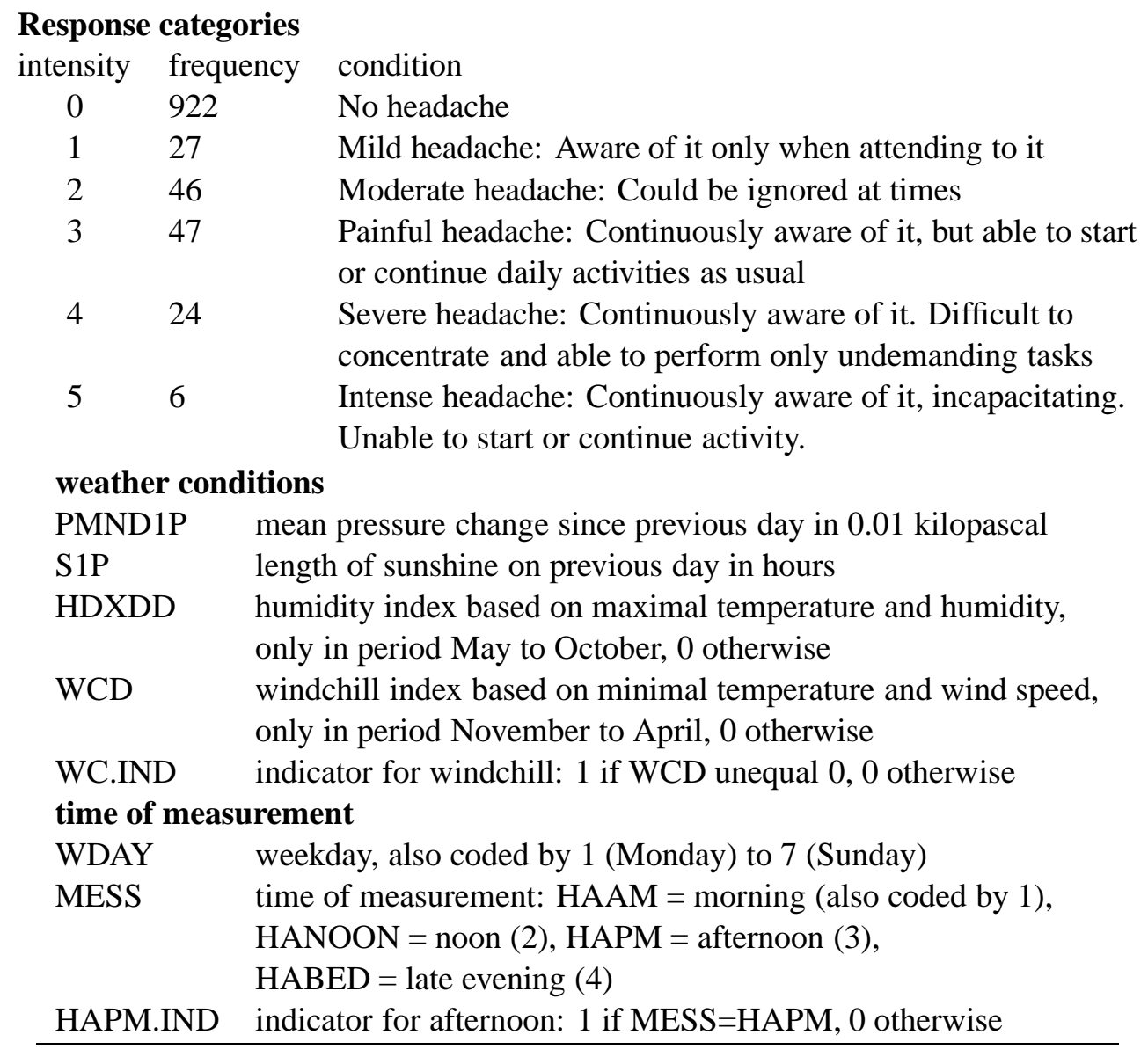



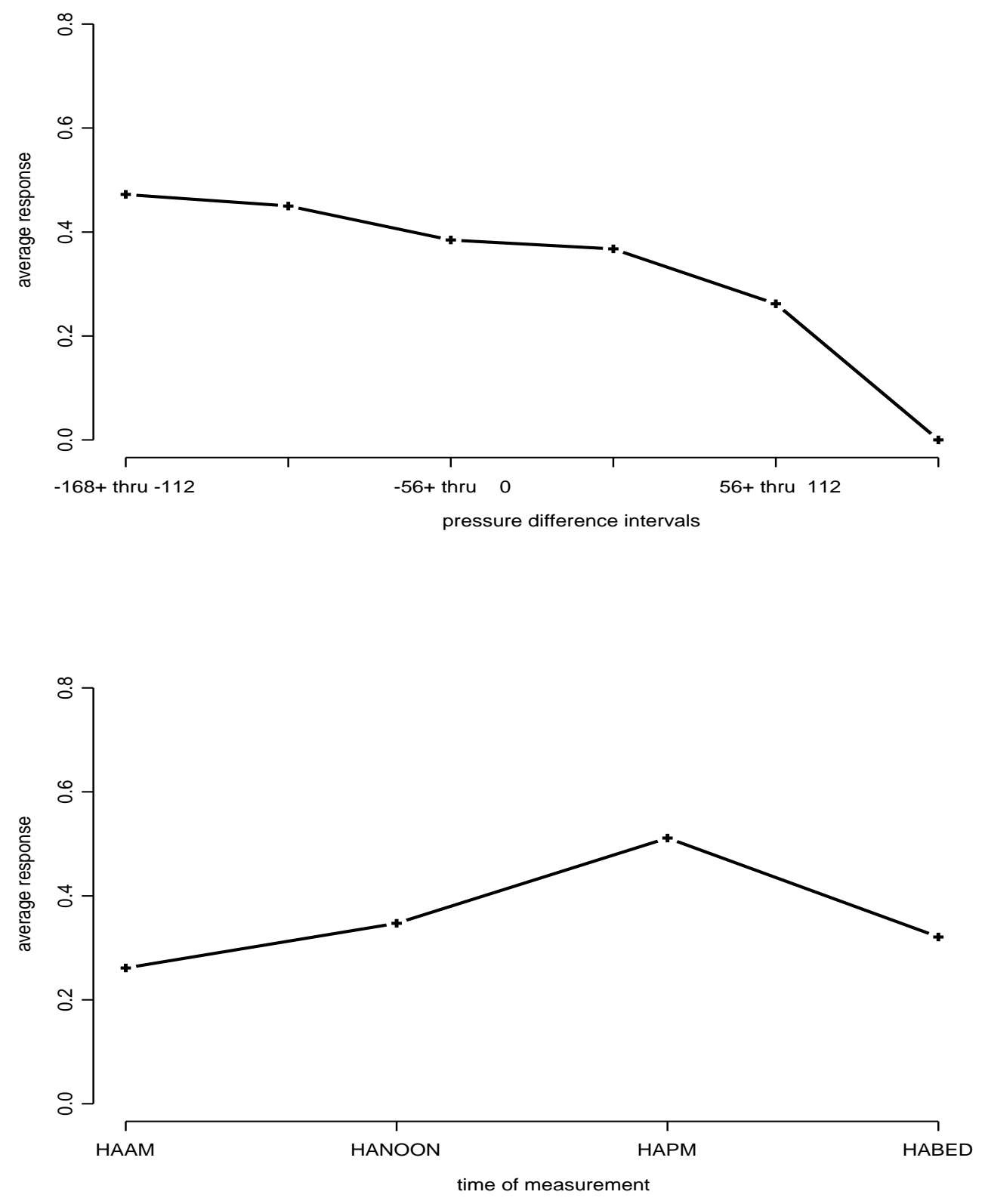

Figure 1: Relationship between average response and pressure difference intervals (top panel) and average response and time of measurement (bottom panel). 
Table 2: Maximum likelihood estimates of regression parameters and cutpoint parameters, residual deviances and Brier scores using the proportional odds model ignoring dependence.

\begin{tabular}{|c|c|c|c|}
\hline & Model A & Model B & Model C \\
\hline \multicolumn{4}{|l|}{ weather conditions } \\
\hline $\mathrm{HDXDD}_{t}$ & -0.4592 & -0.4513 & -0.4011 \\
\hline $\operatorname{HDXDD}_{t}^{2}$ & 0.0109 & 0.0106 & 0.0097 \\
\hline $\mathrm{S}_{1} \mathrm{P}_{t}$ & -0.1055 & -0.1205 & -0.0651 \\
\hline WC.IND $t$ & -4.6821 & -4.7190 & -4.3610 \\
\hline $\begin{array}{l}\text { PMND1P } \\
\text { time of measurement }\end{array}$ & 0.0035 & -0.0149 & -0.0147 \\
\hline HAPM.IND $_{t}$ & -0.4719 & -0.5051 & -0.5433 \\
\hline TUEWED $_{t}$ & 0.5298 & & \\
\hline TUESUN $_{t}$ & & -0.2180 & -1.0196 \\
\hline WEDFRI $_{t}$ & & -0.2542 & 1.9105 \\
\hline THUSAT $_{t}$ & & -0.3935 & -0.5628 \\
\hline \multicolumn{4}{|l|}{ interactions } \\
\hline $\mathrm{PMND} \mathrm{P}_{t} \cdot \mathrm{TUESUN}_{t}$ & & 0.0150 & 0.0174 \\
\hline $\mathrm{PMND} \mathrm{P}_{t} \cdot \mathrm{WEDFRI}_{t}$ & & 0.0284 & 0.0297 \\
\hline $\mathrm{PMND}_{t} \cdot \mathrm{THUSAT}_{t}$ & & 0.0185 & 0.0188 \\
\hline $\mathrm{S} \mathrm{P}_{t} \cdot \mathrm{TUESUN}_{t}$ & & & 0.0703 \\
\hline $\mathrm{S}_{1} \mathrm{P}_{t} \cdot \mathrm{WEDFRI}_{t}$ & & & -0.2218 \\
\hline 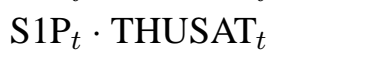 & & & -0.0413 \\
\hline WC.IND $_{t} \cdot$ TUESUN $_{t}$ & & & 0.5248 \\
\hline $\mathrm{WC}^{\mathrm{IND}} t \cdot \mathrm{WEDFRI}_{t}$ & & & -0.9426 \\
\hline $\begin{array}{l}\text { WC.IND }_{t} \cdot \mathrm{THUSAT}_{t} \\
\text { cutpoints }\end{array}$ & & & 1.3245 \\
\hline$\alpha_{0}$ & 6.8128 & 7.3810 & 6.5040 \\
\hline$\alpha_{1}$ & 7.0478 & 7.6272 & 6.7591 \\
\hline$\alpha_{2}$ & 7.6310 & 8.2314 & 7.3874 \\
\hline$\alpha_{3}$ & 8.6903 & 9.3101 & 8.5073 \\
\hline$\alpha_{4}$ & 10.2024 & 10.8279 & 10.0509 \\
\hline residual deviance (df) & $1106(4753)$ & $1083(4748)$ & $1056(4742)$ \\
\hline Brier score & .2545 & .2467 & .2405 \\
\hline
\end{tabular}


Table 3: Posterior mean and quantile estimates for standardized regression parameters and cutpoint parameters using the AOP model and their deviance information criterion and Brier score

\begin{tabular}{|c|c|c|c|c|c|c|}
\hline & \multicolumn{3}{|c|}{ Model I } & \multicolumn{3}{|c|}{ Model II } \\
\hline & $2.5 \%$ & mean & $97.5 \%$ & $2.5 \%$ & mean & $97.5 \%$ \\
\hline intercept & 0.8817 & 1.2969 & 1.7624 & 1.0610 & 1.4764 & 1.9456 \\
\hline \multicolumn{7}{|c|}{ weather conditions } \\
\hline $\mathrm{HDXDD}_{t}^{s}$ & -2.3685 & -1.2880 & -0.3530 & -2.3874 & -1.3548 & -0.4171 \\
\hline$\left(\operatorname{HDXDD}_{t}^{2}\right)^{s}$ & 0.4096 & 1.1552 & 2.0173 & 0.4616 & 1.2054 & 2.0311 \\
\hline $\mathrm{S} 1 \mathrm{P}_{t}^{s}$ & -0.2368 & -0.1322 & -0.0314 & -0.2688 & -0.1619 & -0.0569 \\
\hline WC.IND $_{t}$ & -1.6215 & -0.8410 & -0.1464 & -1.6499 & -0.8959 & -0.2006 \\
\hline $\mathrm{PMND}_{1} \mathrm{P}_{t}^{s}$ & & & & -0.1331 & -0.0172 & 0.0937 \\
\hline \multicolumn{7}{|c|}{ time of measurement } \\
\hline HAPM.IND $_{t}$ & -0.9163 & -0.5924 & -0.2612 & -0.9194 & -0.5769 & -0.2469 \\
\hline WEDFRI $_{t}$ & & & & -0.2672 & -0.0079 & 0.2609 \\
\hline THUSAT $_{t}$ & & & & -0.5213 & -0.2899 & -0.0535 \\
\hline \multicolumn{7}{|l|}{ interactions } \\
\hline \multicolumn{7}{|l|}{$\mathrm{PMND}_{1} \mathrm{P}_{t}^{s}$} \\
\hline \multicolumn{7}{|c|}{ autoregressive parameter } \\
\hline$\phi$ & 0.7404 & 0.8077 & 0.8718 & 0.7250 & 0.7932 & 0.8541 \\
\hline \multicolumn{7}{|l|}{ cutpoints } \\
\hline$\alpha_{1}$ & 0.4706 & 0.7314 & 1.0221 & 0.4596 & 0.7383 & 1.1732 \\
\hline$\alpha_{2}$ & 1.0821 & 1.3851 & 1.6962 & 1.1002 & 1.4021 & 1.8384 \\
\hline$\alpha_{3}$ & 1.5870 & 1.8979 & 2.2151 & 1.6049 & 1.9250 & 2.3588 \\
\hline$\alpha_{4}$ & 1.8548 & 2.1704 & 2.5127 & 1.8644 & 2.2013 & 2.6321 \\
\hline \multicolumn{7}{|c|}{ deviance information criterion } \\
\hline & $\overline{D(\theta)}$ & $p_{D}$ & DIC & $\overline{D(\theta)}$ & $p_{D}$ & DIC \\
\hline & 799.6967 & 97.8068 & 897.5035 & 787.8536 & 92.5850 & 880.4387 \\
\hline \multicolumn{7}{|l|}{ Bayes factor } \\
\hline $\log \left(f\left(\boldsymbol{y} \mid \boldsymbol{\theta}^{o}, M\right)\right)$ & & & -417.5238 & & & -407.8493 \\
\hline $\log \left(p\left(\boldsymbol{\theta}^{o} \mid M\right)\right)$ & & & -26.6353 & & & -39.5151 \\
\hline $\log \left(p\left(\boldsymbol{\theta}^{o} \mid \boldsymbol{y}, M\right)\right)$ & & & 16.8070 & & & 22.7356 \\
\hline $\log (m(\boldsymbol{y} \mid M))$ & & & -460.9661 & & & -470.1000 \\
\hline
\end{tabular}

Bayes Factor of Model I versus Model II $=\exp (-460.9661+470.1000)=\mathbf{9} \mathbf{2 6 4 . 0 8}$ Brier score 


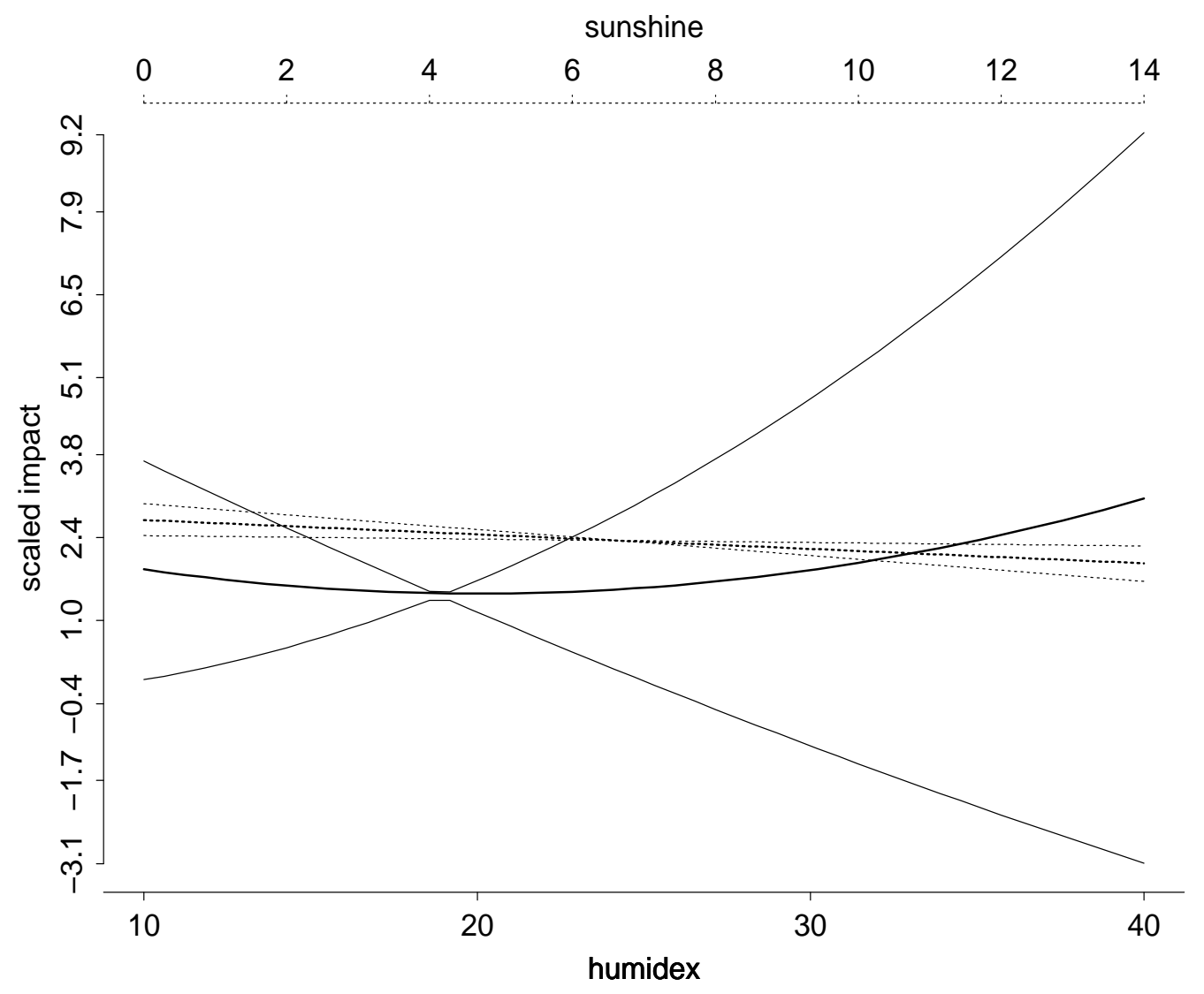

Figure 2: Scaled Impacts for humidex (solid) and sunshine (dotted) with 95\% CI's

Table 4: Posterior mean and quantile estimates of the cumulative odds changes for AOP Model I

\begin{tabular}{rrrr} 
cumulative odds change & $2.5 \%$ & mean & $97.5 \%$ \\
\hline humidex from 10 to 20 & 0.08 & 0.66 & 5.31 \\
humidex from 20 to 30 & 0.10 & 1.49 & 21.73 \\
humidex from 30 to 40 & 0.14 & 3.34 & 88.99 \\
humidex from 40 to 50 & 0.18 & 7.48 & 364.19 \\
humidex from 20 to 40 & 0.01 & 4.97 & 1934.80 \\
\hline $2 \mathrm{hr}$ more sunshine & 1.02 & 1.11 & 1.21 \\
$5 \mathrm{hr}$ more sunshine & 1.06 & 1.30 & 1.60 \\
$10 \mathrm{hr}$ more sunshine & 1.13 & 1.69 & 2.54 \\
\hline Windchill from present & & & \\
to absent & 1.30 & 4.60 & 18.94 \\
\hline PM measurement to & & & \\
no PM measurement & 1.60 & 2.93 & 5.27 \\
\hline
\end{tabular}

